\title{
Inflight performance and calibration of the NuSTAR CdZnTe pixel detectors
}

\author{
Takao Kitaguchi ${ }^{a}$, Varun Bhalerao ${ }^{b}$, W. Rick Cook $^{c}$, Karl Forster ${ }^{c}$, Brian W. Grefenstette ${ }^{c}$, \\ Fiona A. Harrison ${ }^{c}$, Kristin K. Madsen ${ }^{c}$, Peter H. Mao ${ }^{c}$, Hiromasa Miyasaka ${ }^{c}$, \\ Vikram R. Rana ${ }^{c}$, and the NuSTAR team \\ ${ }^{a}$ RIKEN Nishina Center, 2-1 Hirosawa, Wako, Saitama 351-0198, Japan; \\ ${ }^{b}$ Inter-University Center for Astronomy and Astrophysics, Post Bag 4, Ganeshkhind, \\ Pune 411007, India; \\ ${ }^{c}$ Cahill Center for Astronomy and Astrophysics, California Institute of Technology, Pasadena, \\ CA 91125, USA;
}

\begin{abstract}
The Nuclear Spectroscopic Telescope Array (NuSTAR) satellite is a NASA Small Explorer mission designed to operate the first focusing high-energy X-ray (3-79 keV) telescope in orbit. Since the launch in June 2012, all the NuSTAR components have been working normally. The focal plane module is equipped with an ${ }^{155} \mathrm{Eu}$ radioactive source to irradiate the CdZnTe pixel detectors for independent calibration separately from optics. The inflight spectral calibration of the CdZnTe detectors is performed with the onboard ${ }^{155} \mathrm{Eu}$ source. The derived detector performance agrees well with ground-measured data. The in-orbit detector background rate is stable and the lowest among past high-energy X-ray instruments.
\end{abstract}

Keywords: NuSTAR, CdZnTe, High-energy X-rays

\section{INTRODUCTION}

High-energy (> $10 \mathrm{keV})$ X-rays carry fruitful astrophysical information such as super-hot thermal plasma emission, non-thermal emission from accelerated particles, and emission lines following nuclear decay. However, it is relatively difficult to achieve high sensitivity in this energy band, compared to soft $(<10 \mathrm{keV}) \mathrm{X}$-ray observations. This is because high-energy X-ray focusing based on total external reflection, which is used for concentrating soft $\mathrm{X}$-rays, requires a very small incident angle of $<1$ degree, and therefore makes the focal length long and the field of view narrow. Another difficulty with respect to X-ray detectors on the focal plane is that the dominant physical interaction of high-energy X-rays with matter shifts from photoelectric absorption to Compton scattering which makes it difficult to determine the incident X-ray energy unless all scattered X-rays are detected. Furthermore, signal fluxes from astronomical objects generally decrease with energy, while the detector background, mainly including radioactivation background induced by protons trapped in the South Atlantic Anomaly (SAA), is flat. Thus the signal-to-noise ratio gets worse at higher energies.

The Nuclear Spectroscopic Telescope Array $\left(N u S T A R^{1}\right)$ mission, successfully launched on 2012 June 13, is one of the NASA Small Explorer program series. NuSTAR carries the first focusing high-energy X-ray telescope in near-equatorial (6 degree inclination), low-Earth ( $600 \mathrm{~km}$ altitude) orbit and observes astronomical objects in the 3-79 keV energy band. NUSTAR has two sets of identical co-aligned telescopes with depth-graded multilayer optics $^{2}$ based on Bragg reflection and cadmium zinc telluride (CdZnTe or CZT) pixel detectors. ${ }^{3}$ Owing to the focusing optics and position-sensitive detectors, NuSTAR achieves the highest sensitivity ever observed above $10 \mathrm{keV}$, and it has the capability to detect high-energy X-ray point sources with a flux down to sub $\mu$ Crab given a long enough exposure time.

In this paper, we present the inflight performance and calibration of the $N u S T A R$ focal plane detectors. Section 2 gives an overview of the focal plane detectors, the offline data processing, and the detector operation. In Section 3, we describe inflight detector calibration with X-ray irradiation using an onboard radioactive source.

Further author information: (Send correspondence to T.K.) T.K.: E-mail: kitaguti@crab.riken.jp

Space Telescopes and Instrumentation 2014: Ultraviolet to Gamma Ray, edited by Tadayuki Takahashi, Jan-Willem A. den Herder, Mark Bautz, Proc. of SPIE Vol. 9144, 91441R · (C) 2014 SPIE CCC code: 0277-786X/14/\$18 - doi: 10.1117/12.2057342

Proc. of SPIE Vol. $914491441 \mathrm{R}-1$ 
The in-orbit background and its reduction methods are explained in Section 4. Details of pre-flight measurements and spectral modeling of the CdZnTe pixel detectors are described elsewhere. ${ }^{4}$ An overview of the $N u S T A R$ satellite and its scientific highlights in the first two years since the launch can be found in Madsen et al. 2014. ${ }^{5}$ A detailed description of the in-orbit optics performance mainly dealing with the point spread function can be found in An et al. 2014. ${ }^{6}$

\section{OVERVIEW OF FOCAL PLANE DETECTORS}

NuSTAR consists of two identical focal plane modules named FPMA and FPMB, each module having four CdZnTe hybrid pixel detectors (DET0, DET1, DET2, and DET3) arranged in a two-by-two array. The detector array is covered by a cylindrical active shield made of a cesium iodide (CsI) crystal which is coupled with a photomultiplier tube (PMT) to generate a veto signal and discard the detector background with anti-coincidence. Each CdZnTe detector has an active collecting area of $2 \mathrm{~cm} \times 2 \mathrm{~cm}$ and a thickness of $\sim 2 \mathrm{~mm}$. The cathode electrode is a monolithic platinum contact, while the anode electrode is segmented into a $32 \times 32$ grid with a pixel pitch of $605 \mu \mathrm{m}$. The custom $\mathrm{ASIC}^{7}$ of each detector contains a common analog-to-digital converter and 1024 channels of preamplifier, shaping amplifier, sample and hold circuit, and discriminator to read out a signal of the individual anode pixels. An onboard processor identifies the pixel with the largest pulse height and records pulse height information of a three-by-three pixel array consisting of it and eight neighbors.

In the offline analysis software, NuSTARDAS*, NuSTAR events are graded based on charge split morphology in the three-by-three pixel array with a software threshold of $2 \mathrm{keV}$. Next, the graded events of each pixel are processed with the corresponding calibration database ${ }^{\dagger}$. Last, the calibrated nine-pixel pulse heights are used to reconstruct an incident X-ray energy and a three-dimensional position of X-ray interaction. The estimated interaction depth is used to reduce internal detector background particularly produced by radioactivation as well as low-energy spectral tailing due to hole trapping in CdZnTe.

The focal plane detectors were powered up on 2012 June 22, 9 days after the launch. All components have been working properly since then. Detector temperatures of FPMA and FPMB are controlled at 3 and $5^{\circ} \mathrm{C}$, respectively. High voltages applied to the detectors of FPMA and FPMB are -400 and $-450 \mathrm{~V}$, respectively. The surrounding CsI shields of FPMA and FPMB has operated with PMT voltages of 1135 and 1064 V, respectively, so that the energy threshold to generate a veto signal for anti-coincidence rejection is $\sim 40 \mathrm{keV}$. These setting values are the same as those in on-ground calibration measurements to give the best performance.

\section{IN-ORBIT CALIBRATION AND PERFORMANCE}

\subsection{Onboard Calibration Source}

Each focal plane detector is equipped with an ${ }^{155} \mathrm{Eu}$ radioactive source, which emits several X-ray lines in energy range of $6-105 \mathrm{keV}$ with a half-life of 4.8 years and radioactivity of $\sim 10 \mu \mathrm{Ci}$ as of the launch date. The radioactive source is located on a retractable arm that can move the source into and out of the the field of view of the detectors. When the source is deployed, it evenly illuminates all of the detectors. The source enables independent calibration of the focal plane detectors separately from the optics. Before the launch, strong X-ray line intensities of the ${ }^{155} \mathrm{Eu}$ source were well calibrated with an accuracy of $<3 \%$ by using both Si and Ge detectors which were previously cross-calibrated with radioactive sources of ${ }^{55} \mathrm{Fe},{ }^{57} \mathrm{Co}$, and ${ }^{241} \mathrm{Am}$.

The inflight ${ }^{155} \mathrm{Eu}$ spectrum in Figure 1 shows a sharp spectral response to X-ray lines. The FWHM energy resolution below $50 \mathrm{keV}$ is $0.4 \mathrm{keV}$, limited by the electronics noise, while at high energies the energy resolution gradually increases to $1 \mathrm{keV}$ at $86 \mathrm{keV}$. The energy resolution broadens both due to Poisson noise in the number of charge carriers in the CdZnTe as well as increase in the low-energy tail caused by photons interacting closer to the anode (which we refer to as "deeper" in the detector). The inflight spectral response to these X-ray lines is well consistent with pre-launch calibration measurements.

\footnotetext{
${ }^{*}$ NuSTARDAS is freely available as a package of HEASoft: https://heasarc.gsfc.nasa.gov/lheasoft/

${ }^{\dagger}$ http://heasarc.gsfc.nasa.gov/docs/heasarc/caldb/nustar/
} 


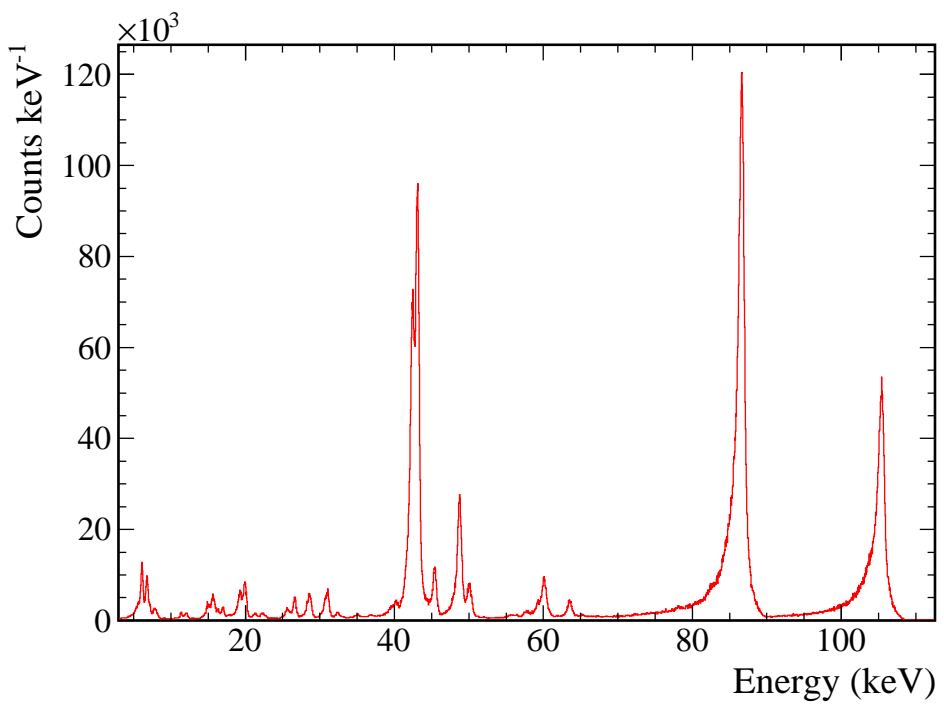

Figure 1. Inflight spectrum of the FPMB/DET0 detector irradiated with the onboard ${ }^{155}$ Eu radioactive source. The energy resolution in FWHM is $0.4 \mathrm{keV}$ below $50 \mathrm{keV}$ and $1.0 \mathrm{keV}$ at $86 \mathrm{keV}$.

\subsection{Spectral Response}

As mentioned in $\S 3.1$, the inflight spectral response measured with the the ${ }^{155} \mathrm{Eu}$ source matches well with ground-measured data. Therefore, the customized Monte-Carlo simulation program ${ }^{4}$ with calibration database determined with on-ground measurements has been used to model the spectral response function.

The on-ground calibration measurements show that each pixel has different mobility-lifetime products of electrons and holes. ${ }^{4}$ These values of each CdZnTe detector scatter by a factor of 2 with a modestly strong correlation between electrons and holes. However, the measured data and the Monte-Carlo simulation show that the spectral response is little affected by the difference of the charge transport properties. Thus, the spectral response function of each $\mathrm{CdZnTe}$ detector is modeled with the average of mobility-lifetime products under the assumption that the spectral response is uniform across the detector to simplify the spectral analysis. On the other hand, actual observed events are processed with the pixel-by-pixel calibration database so that the variability of pixel properties is cancelled out.

In Figure 2, the ${ }^{155}$ Eu spectrum of an isolated nuclear $\gamma$-ray at $60 \mathrm{keV}$, which is near the energy of astronomical $\gamma$-rays emitted following ${ }^{44} \mathrm{Ti}$ decay, is compared with the spectral model generated by a convolution of a narrow Gaussian line with the spectral response function. The spectral model, which includes the low-energy tail structure due to hole trapping in $\mathrm{CdZnTe}$, is in good agreement with the measured data.

\subsection{Energy Scale}

Before the launch, the pixel-by-pixel energy scale calibration for each event grade was performed by individually irradiating the hybrid detector with two radioactive sources of ${ }^{241} \mathrm{Am}$ and inflight ${ }^{155} \mathrm{Eu}$. The calibration data show a good linearity of the energy scale with no significant non-linearity. After the launch, the energy scale was determined with the onboard ${ }^{155} \mathrm{Eu}$ source in the same way as the pre-launch measurements and was found to slightly change. Therefore, the pre-launch energy scale was tuned without non-linearity so that the resulting energy scale accuracy is typically $<20 \mathrm{eV}$ in $6-80 \mathrm{keV}$.

Below $6 \mathrm{keV}$ where there is no available line for calibration, the energy scale is linearly extrapolated. In order to verify the energy scale in this band, we use fluorescence X-rays from the Cassiopeia A (Cas A) supernova remnant. We compared the line energies that we obtain with NuSTAR to the results we obtained with Suzaku/XIS, which is an X-ray CCD camera with an accurate energy determination of $\lesssim 10 \mathrm{eV} .{ }^{8}$ The observed spectra of NuSTAR and Suzaku/XIS in Figure 3 were individually fitted by a continuum model consisting of 


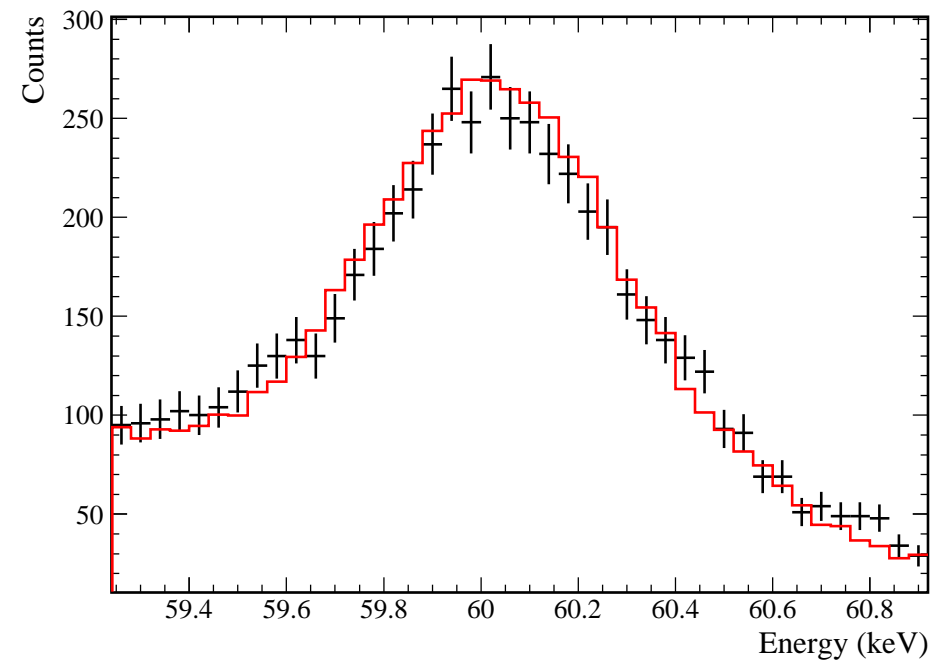

Figure 2. Spectral comparison of the $60 \mathrm{keV}$ line between (red) model and (black) actual FPMB/DET0 data obtained with the onboard ${ }^{155} \mathrm{Eu}$ source.

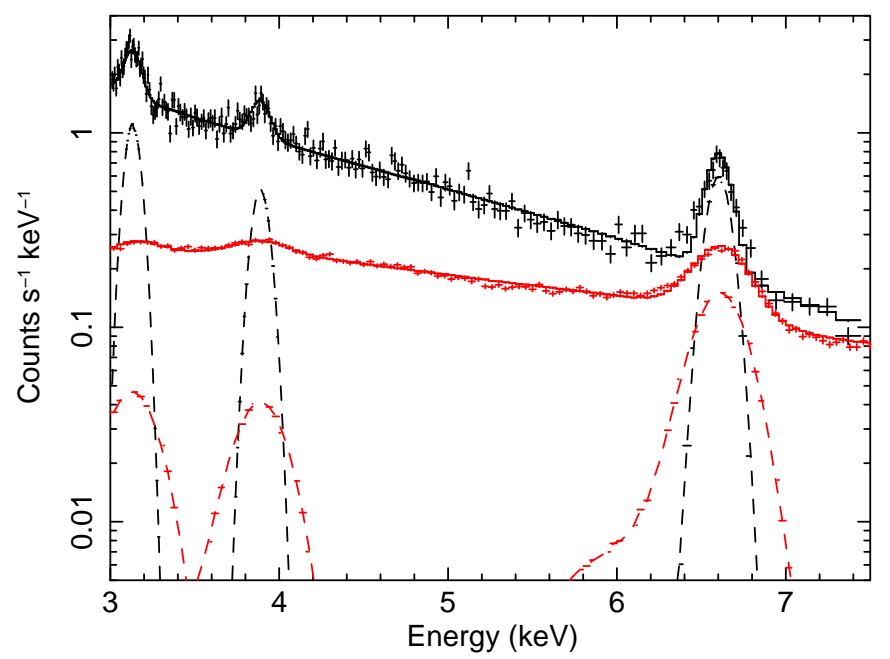

Table 1. Line center energies of the best-fit Gaussian models to the Cas A spectra in Figure 3.

\begin{tabular}{ccc}
\hline \hline & \multicolumn{2}{c}{ Line center energy $(\mathrm{keV})$} \\
& NuSTAR & Suzaku \\
\hline $\mathrm{Ar} \mathrm{K}$ & $3.130 \pm 0.016$ & $3.128_{-0.004}^{+0.006}$ \\
$\mathrm{Ca} \mathrm{K}$ & $3.886 \pm 0.017$ & $3.890_{-0.008}^{+0.010}$ \\
$\mathrm{Fe} \mathrm{K}$ & $6.621 \pm 0.004$ & $6.606_{-0.006}^{+0.007}$ \\
\hline
\end{tabular}

Figure 3. Cas A spectra observed with (red) NuSTAR/FPMB and (black) Suzaku/XIS-3FI. The dashed lines show the bestfit Gaussian models. These spectra are extracted from the $\mathrm{Ca} / \mathrm{Fe}-$ rich western region of Cas A.

a thermal Bremsstrahlung and power-law emission with three narrow Gaussian lines, each corresponding to Ar $\mathrm{K}_{\alpha}$, Ca $\mathrm{K}_{\alpha}$, and $\mathrm{Fe} \mathrm{K}_{\alpha}$. The best-fit line center energies listed in Table 1 show that the $N u S T A R$ energy scale accuracy in the lower-energy band is $<20 \mathrm{eV}$.

\section{IN-ORBIT BACKGROUND}

The NuSTAR focal plane detector mainly has two methods of discarding background events. The CsI scintillator surrounding the detector array can not only actively shield charged particles with on-board anti-coincidence, but also passively shield low-energy $\gamma$-rays. Furthermore, the interaction depth estimated in the offline software can 


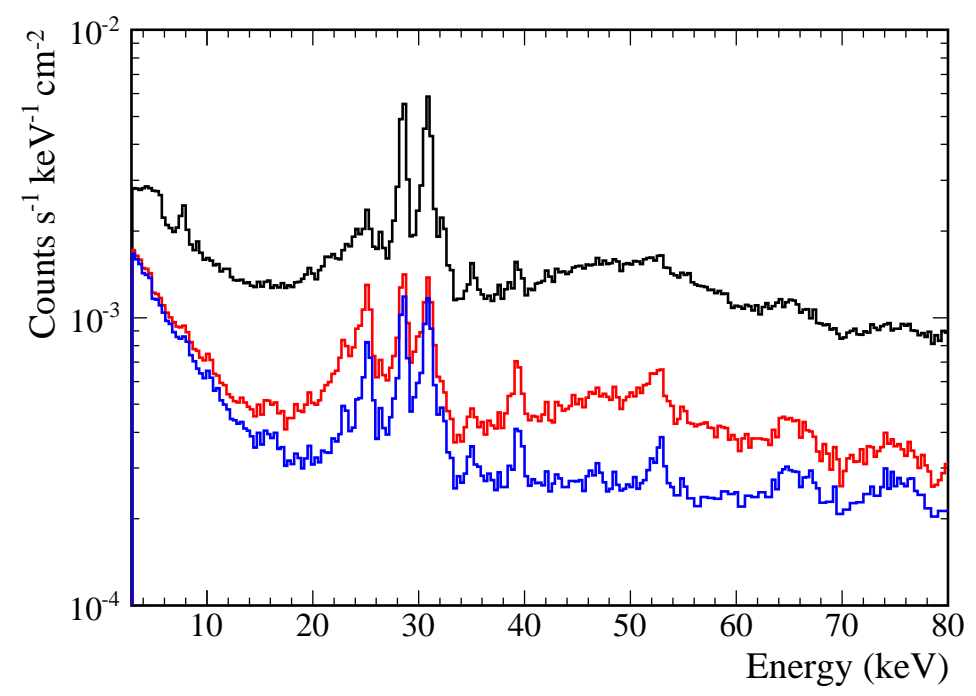

Figure 4. Comparison of in-orbit background spectra normalized by the geometrical area with different background rejection methods. The black, red, and blue spectra shows background spectra without any rejection, with anti-coincidence, and with all background rejection including the interaction depth cut, respectively. The data were obtained from observations of a blank sky, the North Ecliptic Pole.

reduce activation background, about half of which is more deeply distributed in the CdZnTe detector than X-ray events.

A comparison of background spectra in Figure 4 shows the effectiveness of the rejection methods. After applying all background cuts, the background level decreases by about one order of magnitude. The two background lines at 29 and $31 \mathrm{keV}$ originate from the fluorescence X-rays of I and Cs from the shield, respectively. The other lines are produced by detector activation, and are distributed uniformly throughout the detector. This source of background is efficiently reduced by the interaction depth cut. On the other hand, background signals in the low-energy band $(<10 \mathrm{keV})$ are mainly produced by the cosmic X-ray background passing through the optics or the detector aperture, thus making the depth cut ineffective for distinguishing them from valid X-ray events.

The final background rates in a half power diameter of the focal spot are $1 \times 10^{-3}$ and $8 \times 10^{-4}$ counts s$^{-1}$ in the energy ranges of 10-30 and 30-60 keV, respectively. They are in a good agreement with a pre-launch estimation and meet the design requirement. These background rates are more than two orders of magnitude lower than that achieved by collimated detectors (e.g. sub counts $\mathrm{s}^{-1}$ for Suzaku/HXD-PIN ${ }^{9}$ ). Owing to the near-equatorial orbit where the satellite grazes the SAA and is less exposed to trapped protons, the background spectrum remains largely unchanged since the launch. A more detailed description of the background spectrum and an empirical background modeling by using blank-sky spectra can be found in [10].

\section{SUMMARY}

The NuSTAR focal plane detectors has been working normally since the launch. The in-orbit detector performance obtained with the onboard ${ }^{155} \mathrm{Eu}$ source is well consistent with pre-launch calibration measurements and simulation predictions. The inflight calibration status is summarized as follows:

- The FWHM energy resolution is $0.4 \mathrm{keV}$ below $50 \mathrm{keV}$, and increases to $1.0 \mathrm{keV}$ at $86 \mathrm{keV}$.

- The spectral response model can well reproduce the isolated $60 \mathrm{keV} \gamma$-ray line.

- The energy determination accuracy is $<20 \mathrm{eV}$ in the $3-80 \mathrm{keV}$ energy range. 
- The background rates in a half power diameter of the focal spot are $1 \times 10^{-3}$ and $8 \times 10^{-4}$ counts s$^{-1}$ in the 10-30 and 30-60 keV energy bands, respectively.

\section{ACKNOWLEDGMENTS}

This work was supported under NASA Contract No. NNG08FD60C, and made use of data from the NuSTAR mission, a project led by the California Institute of Technology, managed by the Jet Propulsion Laboratory, and funded by the National Aeronautics and Space Administration. We thank the NuSTAR Operations, Software and Calibration teams for support with the execution and analysis of these observations. This research has made use of the NuSTAR Data Analysis Software (NuSTARDAS) jointly developed by the ASI Science Data Center (ASDC, Italy) and the California Institute of Technology (USA). T.K. was supported by Japan Society for the Promotion of Science (JSPS) Grant-in-Aid for Young Scientists (B) (No. 24740185).

\section{REFERENCES}

[1] Harrison, F. A., Craig, W. W., Christensen, F. E., Hailey, C. J., Zhang, W. W., Boggs, S. E., Stern, D., Cook, W. R., Forster, K., Giommi, P., Grefenstette, B. W., Kim, Y., Kitaguchi, T., Koglin, J. E., Madsen, K. K., Mao, P. H., Miyasaka, H., Mori, K., Perri, M., Pivovaroff, M. J., Puccetti, S., Rana, V. R., Westergaard, N. J., Willis, J., Zoglauer, A., An, H., Bachetti, M., Barrière, N. M., Bellm, E. C., Bhalerao, V., Brejnholt, N. F., Fuerst, F., Liebe, C. C., Markwardt, C. B., Nynka, M., Vogel, J. K., Walton, D. J., Wik, D. R., Alexander, D. M., Cominsky, L. R., Hornschemeier, A. E., Hornstrup, A., Kaspi, V. M., Madejski, G. M., Matt, G., Molendi, S., Smith, D. M., Tomsick, J. A., Ajello, M., Ballantyne, D. R., Baloković, M., Barret, D., Bauer, F. E., Blandford, R. D., Brandt, W. N., Brenneman, L. W., Chiang, J., Chakrabarty, D., Chenevez, J., Comastri, A., Dufour, F., Elvis, M., Fabian, A. C., Farrah, D., Fryer, C. L., Gotthelf, E. V., Grindlay, J. E., Helfand, D. J., Krivonos, R., Meier, D. L., Miller, J. M., Natalucci, L., Ogle, P., Ofek, E. O., Ptak, A., Reynolds, S. P., Rigby, J. R., Tagliaferri, G., Thorsett, S. E., Treister, E., and Urry, C. M., "The Nuclear Spectroscopic Telescope Array (NuSTAR) High-energy X-Ray Mission," ApJ 770, 103 (June 2013).

[2] Hailey, C. J., An, H., Blaedel, K. L., Brejnholt, N. F., Christensen, F. E., Craig, W. W., Decker, T. A., Doll, M., Gum, J., Koglin, J. E., Jensen, C. P., Hale, L., Mori, K., Pivovaroff, M. J., Sharpe, M., Stern, M., Tajiri, G., and Zhang, W. W., "The Nuclear Spectroscopic Telescope Array (NuSTAR): optics overview and current status," in [Society of Photo-Optical Instrumentation Engineers (SPIE) Conference Series], Society of Photo-Optical Instrumentation Engineers (SPIE) Conference Series 7732 (July 2010).

[3] Rana, V. R., Cook, W. R., Harrison, F. A., Mao, P. H., and Miyasaka, H., "Development of focal plane detectors for the Nuclear Spectroscopic Telescope Array (NuSTAR) mission," in [Society of Photo-Optical Instrumentation Engineers (SPIE) Conference Series], Society of Photo-Optical Instrumentation Engineers (SPIE) Conference Series $\mathbf{7 4 3 5}$ (Aug. 2009).

[4] Kitaguchi, T., Grefenstette, B. W., Harrison, F. A., Miyasaka, H., Bhalerao, V. B., Cook, W. R., Mao, P. H., Rana, V. R., Boggs, S. E., and Zoglauer, A. C., "Spectral calibration and modeling of the NuSTAR CdZnTe pixel detectors," in [Society of Photo-Optical Instrumentation Engineers (SPIE) Conference Series], Society of Photo-Optical Instrumentation Engineers (SPIE) Conference Series 8145 (Sept. 2011).

[5] Madsen, K. K., Harrison, F. A., Christensen, F. E., Craig, W. W. Hailey, C. J., Zhang, W. W., Boggs, S. E., Stern, D. K., An, H., Cook, W. R., Forster, K., Fuerst, F., Grefenstette, B. W., Kitaguchi, T., Markwardt, C. B., Mao, P. H., Miyasaka, H., Rana, V. R., Zoglauer, A. C., Walton, D. J., and Westergaard, N. J. S., "The nuclear spectroscopic telescope array (NuSTAR) high-energy x-ray mission," in [Society of PhotoOptical Instrumentation Engineers (SPIE) Conference Series], Society of Photo-Optical Instrumentation Engineers (SPIE) Conference Series 9144 (June 2014).

[6] An, H., Madsen, K. K., Westergaard, N. J. S., Koglin, J. E. Boggs, S. E., Christensen, F. E., Craig, W. W., Hailey, C. J., H. F. A., Kaspi, V. M., Stern, D. K., and Zhang, W. W., "PSF calibration of the hard x-ray optics of the Nuclear Spectroscopic telescope Array," in [Society of Photo-Optical Instrumentation Engineers (SPIE) Conference Series], Society of Photo-Optical Instrumentation Engineers (SPIE) Conference Series 9144 (June 2014). 
[7] Harrison, F. A., Cook, W. R., Miyasaka, H., and McLean, R., [Semiconductor radiation detection systems], CRC Press, Boca Raton, FL (2010).

[8] Koyama, K., Hyodo, Y., Inui, T., Nakajima, H., Matsumoto, H., Tsuru, T. G., Takahashi, T., Maeda, Y., Yamazaki, N. Y., Murakami, H., Yamauchi, S., Tsuboi, Y., Senda, A., Kataoka, J., Takahashi, H., Holt, S. S., and Brown, G. V., "Iron and Nickel Line Diagnostics for the Galactic Center Diffuse Emission," PASJ 59, 245-255 (Jan. 2007).

[9] Kokubun, M., Makishima, K., Takahashi, T., Murakami, T., Tashiro, M., Fukazawa, Y., Kamae, T., Madejski, G. M., Nakazawa, K., Yamaoka, K., Terada, Y., Yonetoku, D., Watanabe, S., Tamagawa, T., Mizuno, T., Kubota, A., Isobe, N., Takahashi, I., Sato, G., Takahashi, H., Hong, S., Kawaharada, M., Kawano, N., Mitani, T., Murashima, M., Suzuki, M., Abe, K., Miyawaki, R., Ohno, M., Tanaka, T., Yanagida, T., Itoh, T., Ohnuki, K., Tamura, K.-I., Endo, Y., Hirakuri, S., Hiruta, T., Kitaguchi, T., Kishishita, T., Sugita, S., Takahashi, T., Takeda, S., Enoto, T., Hirasawa, A., Katsuta, J., Matsumura, S., Onda, K., Sato, M., Ushio, M., Ishikawa, S.-N., Murase, K., Odaka, H., Suzuki, M., Yaji, Y., Yamada, S., Yamasaki, T., Yuasa, T., and Hxd Team, "In-Orbit Performance of the Hard X-Ray Detector on Board Suzaku," PASJ 59, 53-76 (Jan. 2007).

[10] Wik, D. R., Hornstrup, A., Molendi, S., Madejski, G., Harrison, F. A., Zoglauer, A., Grefenstette, B. W., Gastaldello, F., Madsen, K. K., Westergaard, N. J., Ferreira, D. D. M., Kitaguchi, T., Pedersen, K., Boggs, S. E., Christensen, F. E., Craig, W. W., Hailey, C. J., Stern, D., and Zhang, W. W., "NuSTAR Observations of the Bullet Cluster: Constraints on Inverse Compton Emission," ArXiv e-prints (Mar. 2014). 